\title{
METALLIC WIRE SUBSTRATE (MWS) MICROSTRIP STRUCTURE: CHARACTERIZATION AND APPLICATION TO A PATCH ANTENNA
}

\author{
J.M. Fernandez ${ }^{(1)}$, M. Sierra-Castañer ${ }^{(1)}$, and C. Caloz ${ }^{(2)}$ \\ ${ }^{(1)}$ Universidad Politécnica de Madrid, E.T.S.I. Telecomunicación, 28040 Madrid, SPAIN \\ Email:jmfdez@gr.ssr.upm.es \\ ${ }^{(2)}$ École Polytechnique de Montréal,Poly-Grames Research Center,H3T 1J4, Montréal, CANADA \\ Email: christophe.caloz@polymtl.ca
}

\begin{abstract}
This paper presents the characterization of the novel metallic wire substrate (MWS) introduced in [1] and its application to a microstrip patch antenna. It is shown numerically that the MWS exhibits artificial magnetodielectric properties with simultaneously enhanced effective permittivity and permeability over a large frequency band. Consequently, due to the higher effective refractive index, the effective wavelength is reduced which implies reduction in the size of the elements printed on the MWS. First, the MWS microstrip line is analyzed and a procedure for its effective constitutive parameters $(\varepsilon, \mu)$ extraction is described and a parametric analysis is carried out to show the range of $\varepsilon, \mu$ values achievable with typical substrates. Then, a patch antenna with reduced size is demonstrated, discussed in terms of efficiency, gain and compared to a homogeneous substrate.
\end{abstract}

\section{INTRODUCTION}

Artificial dielectrics consist of subwavelength conducting obstacles embedded in a homogeneous host medium [2]. After their introduction by Kock in 1948 [3], artificial dielectrics were investigated both theoretically and experimentally by several researchers in the 1950s [4-8].

Recently, a novel metallic wire substrate (MWS) was introduced as an artificial dielectric with specific properties for planar microwave circuits [1,9].These properties include magneto-dielectric characteristics, ie simultaneously enhanced effective permittivity and permeability (para-electric and para-magnetic response). This lead to enhanced effective refractive index or reduction of effective guided wavelength, and consequently provide reduction in the size of the circuits. A similar structure was recently presented in [10]. Other magneto-dielectric substrates were discussed by Mossallaei in $[11,12]$; these substrate exhibit interesting characteristics at the expense of the drawbacks of narrow bandwidth (due to their resonant nature), and complex, bulky non-MMIC compatible architecture.

This paper presents a parametric analysis of the metallic wire substrate (MWS) microstrip and its application to a microstrip patch antenna with reduced size with consideration efficiency.

\section{METALLIC WIRE SUBSTRATE (MWS) MICROSTRIP TRANSMISSION LINE}

\subsection{Description of the structure}

Fig. 1(a) shows a microstrip transmission line (TL) printed on a metallic wire substrate (MWS). This MWS is constituted of a dense mesh of metallic wires in a conventional homogeneous substrate. The metallic wires are copper plated via holes with interspacing much smaller than the guided wavelength $\lambda_{g}$, so as to build an effective medium. In order to prevent shortcircuiting contacts, a thin isolating substrate layer is placed between the TL and the MWS as shown in Fig. 1(b).

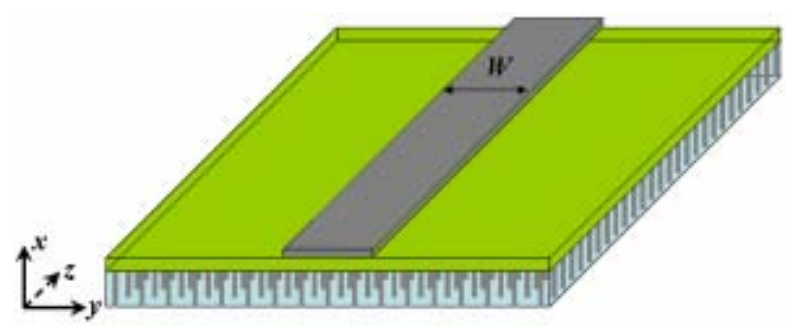

(a) Perspective view.

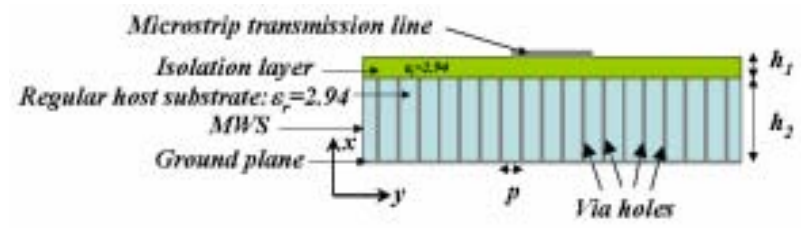

(b) Profile view.

Figure 1. Metallic wire substrate (MWS) microstrip transmission line.

The MWS prototype is shown in Fig. 2(a). The host substrate of the array of metallic wires is Duroid 6002 with dielectric constant $\varepsilon_{\mathrm{r}}$ of 2.94 , loss tangent $\tan \delta$ of 0.0012 at $10 \mathrm{GHz}$ and thickness $h_{2}$ of $0.508 \mathrm{~mm}$. The wire mesh is made of copper plated via holes having diameter $d$ of $0.381 \mathrm{~mm}$ using a laser-drilling and holes plating technology available at the Poly-Grames 
Research Center (www.grames.polymtl.ca). The spacing between adjacent via holes is of $0.635 \mathrm{~mm}$ (center to center). The MWS contains an array of $200 \times 200$ via holes. The backplane of the MWS is also copper plated to create a ground plane. The thin isolating substrate is also Duroid 6002 with thickness $h_{l}$ of $0.508 \mathrm{~mm}$.

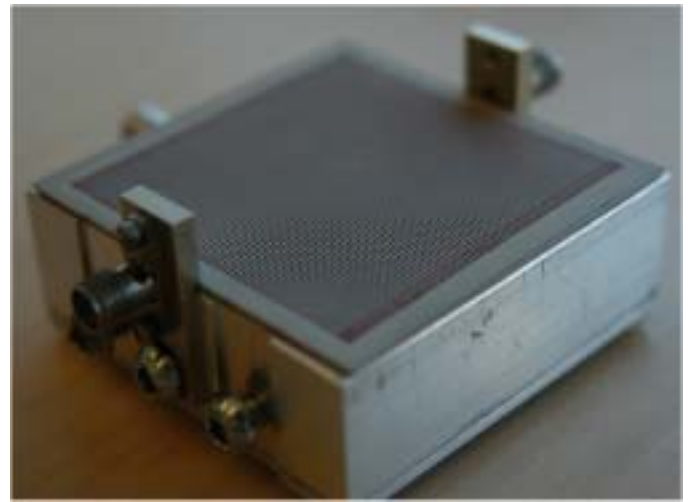

(a) Metallic wire substrate (MWS).

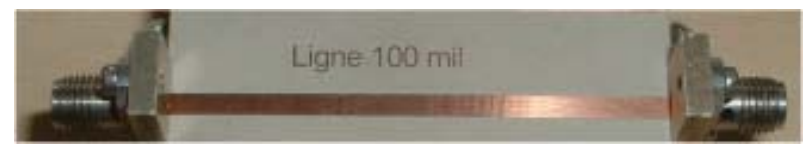

(b) Top view: MWS microstrip line.

Figure 2. MWS microstrip transmission line (TL) prototype.

As shown in Fig. 2(b), a microstrip transmission line (TL) with a width of $2.54 \mathrm{~mm}$ for $50 \Omega$ characteristic impedance and a length of $48 \mathrm{~mm}$ is etched on the isolation layer. This substrate is necessary to prevent short circuit between the TL and the ground.

\subsection{S-parameters}

The MWS microstrip TL prototype shown in Fig. 2 was measured and compared to a microstrip TL on a regular Duroid 6002 substrate with the same length and width. The S-parameters of these two TLs from DC to $20 \mathrm{GHz}$ are shown in Fig. 3.

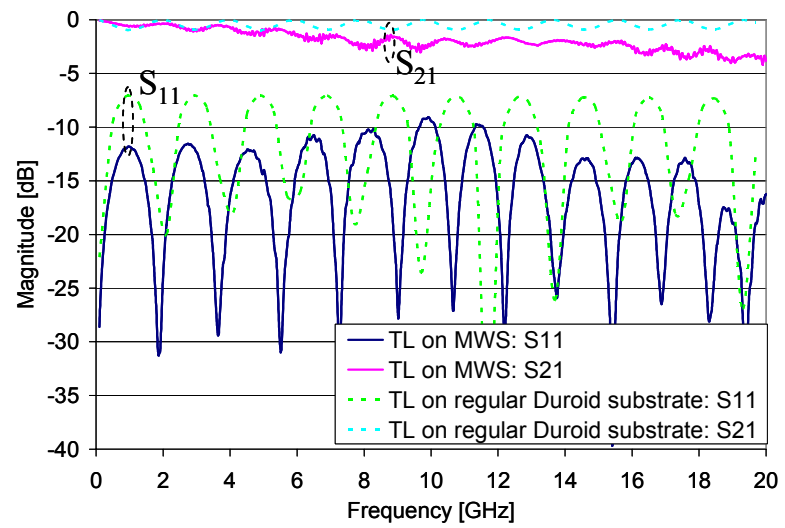

(a) Magnitude.

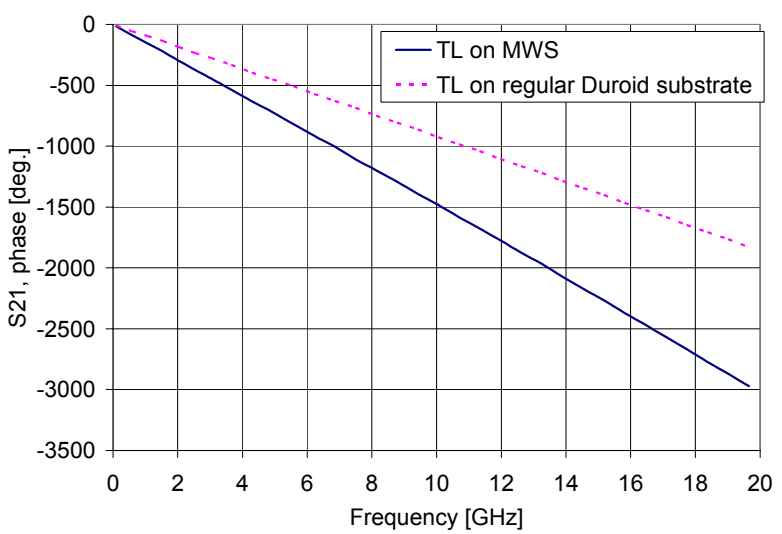

(b) Unwrapped phase.

Figure 3. S-parameters for the metallic wire substrate (MWS) transmission line compared to those of a $50 \Omega$ transmission line on a regular Duroid 6002 substrate.

As shown in Fig. 3(a), good matching (low insertion loss) throughout the measured frequency range is achieved. It may be seen form its larger phase shift that the propagation constant $\beta$ in the MWS structure has been increased compared to its value in the regular Duroid substrate as a consequence of the loading effect of the via. That indicates that the MWS has a higher refractive index $n$ or, equivalently, exhibits slow-wave characteristics.

\subsection{Extraction of effective parameters}

In this section, we extract the transmission line parameters of the microstrip MWS structure to compute its effective parameters $\varepsilon_{\text {eff }}$ and $\mu_{\text {eff. }}$. The propagation constant $\beta$ and input impedance $Z_{\text {in }}$ of a TL are [13]

$$
\begin{gathered}
\beta=-\frac{\theta}{l}=-\frac{\text { unwrapped }\left[\operatorname{phase}\left(S_{21}\right)\right]}{l} \\
Z_{\text {in }}=Z_{0} \frac{Z_{L}+j Z_{0} \tan (\beta l)}{Z_{0}+j Z_{L} \tan (\beta l)}
\end{gathered}
$$

where $Z_{L}=50 \Omega$ is the impedance of the load. By combining (1) and (2) and using the measured Sparameters, the effective propagation constant $\beta$ and characteristic impedance $Z_{0}$ of the MWS TL can be readily obtained.

The propagation constant $\beta$ can also be expressed as a function of the host medium parameters and the free space wave number $k_{0}$ as

$$
\beta=n k_{0}=\sqrt{\varepsilon_{e f f} \mu_{e f f}} k_{0} .
$$


In the most general case of a magneto-dielectric substrate, the formula for the conventional microstrip TL characteristic impedance needs to be generalized to include the contribution of the effective permeability

$$
Z_{0}=\sqrt{\frac{\mu_{\text {eff }}}{\varepsilon_{\text {eff }}}} \frac{120 \pi}{\left\{W /\left(h_{1}+h_{2}\right)+1.393+0.667 \ln \left[W /\left(h_{1}+h_{2}\right)+1.444\right]\right\}}
$$

Combining (3) and (4), the following expressions are obtained for the effective permittivity $\varepsilon_{\text {eff }}$ and permeability $\mu_{e f f}$ after simple algebraic manipulations

$$
\begin{aligned}
& \mu_{\text {eff }}=\xi \frac{Z_{0}}{120 \pi} \frac{\beta}{k_{0}}, \\
& \varepsilon_{\text {eff }}=\frac{1}{\xi} \frac{120 \pi}{Z_{0}} \frac{\beta}{k_{0}},
\end{aligned}
$$

where

$$
\xi=W /\left(h_{1}+h_{2}\right)+1.393+0.667 \ln \left[W /\left(h_{1}+h_{2}\right)+1.444\right]
$$

Fig. 4 shows the effective parameters of the MWS TL prototype compared to those of a simulated $50 \Omega$ transmission line on a regular Duroid 6002 substrate and extracted by the above procedure. The permittivity and the permeability have been enhanced, by a factor of 1.7 [Fig. 4(a)] and 1.3 [Fig. 4(b)] respectively. These enhancements have a concomitant effect in the enhancement of the refractive index.

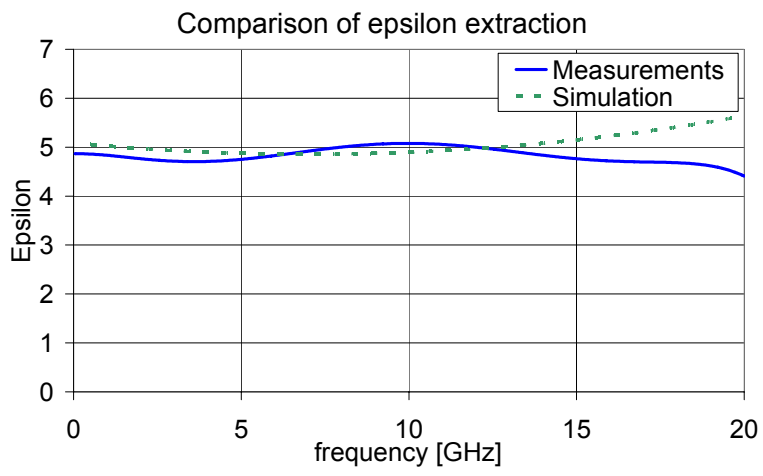

(a) Extracted $\varepsilon_{e f f}$

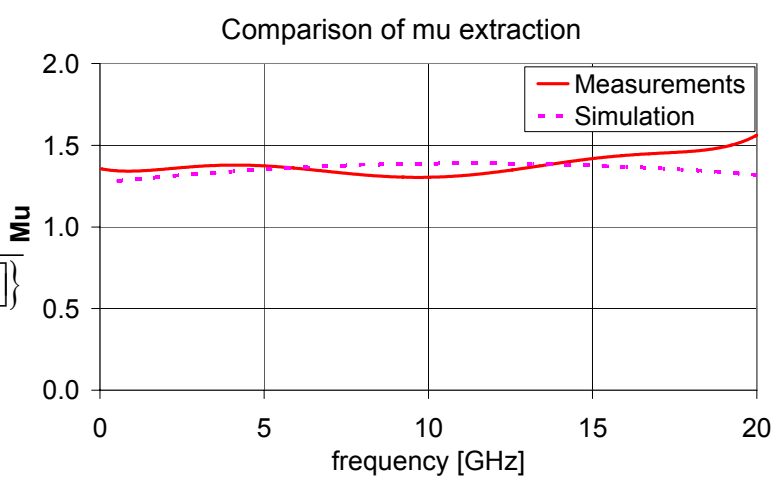

(b) Extracted $\mu_{\text {eff. }}$

Figure 4. Metallic wire substrate (MWS) microstrip transmission line prototype parameters compared to

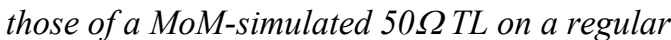
substrate computed by (5) and (6).

Good agreement between the MoM-simulation and the measurements results is observed. The MoM simulation model can therefore be used for the characterization of the metallic wire substrate (MWS), which is carried out next.

\subsection{Parametric characterization}

The MWS microstrip structure is characterized in terms of its effective permittivity and permeability parameters, $\varepsilon_{\text {eff }}$ and $\mu_{\text {eff }}$ versus frequency as a function of the diameter of the wires and of the structured substrate height $h_{2}$, which are the most significant parameters in terms of variations of the $\varepsilon_{\text {eff }}$ and $\mu_{\text {eff. }}$. The results are presented in Fig. 5 and 6, and may be used as charts for designs.

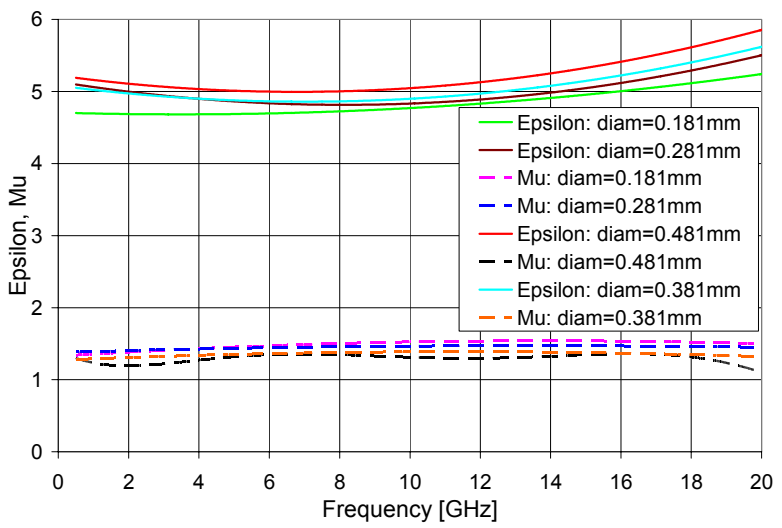

Figure 5. Effect of the variation of via holes diameter on the $\varepsilon_{\text {eff }}$ and $\mu_{\text {eff }}$ parameters. 


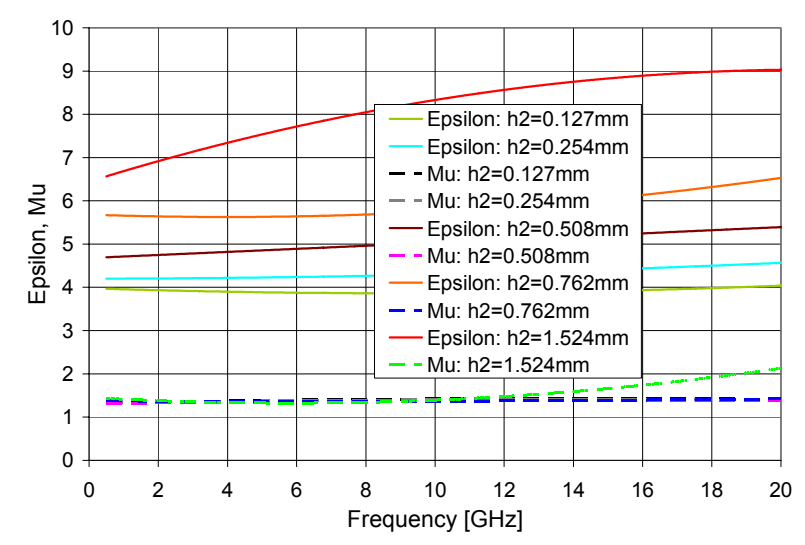

Figure 6. Effect of the variation of substrates thickness on the $\varepsilon_{\text {eff }}$ and $\mu_{\text {eff }}$ parameters.

From a conventional Duroid host substrate with permittivity $\varepsilon_{\mathrm{r}}=2.94$, an MWS's with values of $\varepsilon_{\text {eff }}$ ranging from 4 to 9 and of $\mu_{\text {eff }}$ ranging from 1.3 to 2 have been achieved using parameters that are currently available with the laser-drilling and holes-plating technology at the Poly-Grames Research Center.

The MWS provides novel possibility for the design of planar circuits on substrate with arbitrary permittivity and permeability over a large range of values. In addition, it allows the miniaturization of planar circuits and antennas thanks to its enhanced refractive index compared to that of the host substrate.

\section{APPLICATION: METALLIC WIRE SUBSTRATE (MWS) MICROSTRIP PATCH ANTENNA}

As an application of the MWS presented in the previous section, the inset-fed MWS patch antenna shown in Fig.7(a) was designed and compared with the corresponding microstrip patch antenna on a homogeneous substrate $\left(\varepsilon_{\mathrm{r}}=2.94\right)$ [Fig. $7(\mathrm{~b})$ ] for the same resonant frequency, which has been chosen as 1.9 GHz.

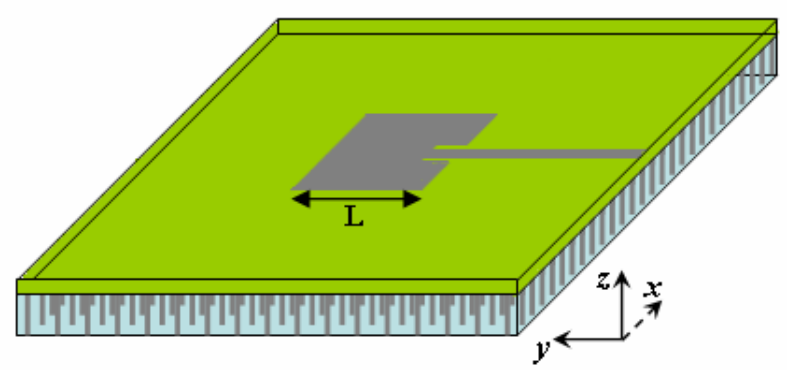

(a) MWS microstrip patch antenna.

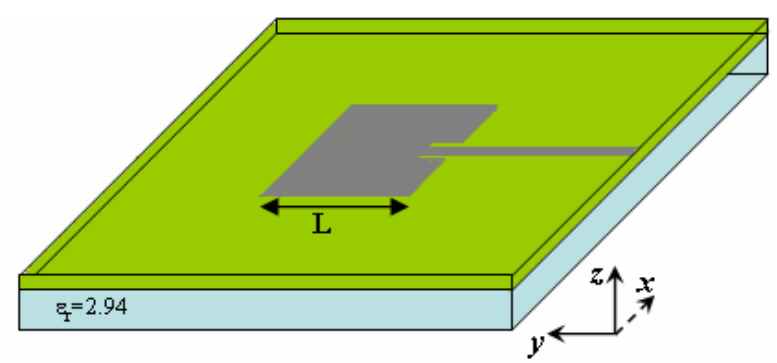

(b)Microstrip patch antenna on a homogeneous substrate $\left(\varepsilon_{r}=2.94\right)$.

Figure 7. Microstrip patch antenna structure.

Since the resonant size of the antenna is $L \cong \lambda_{\mathrm{g}} / 2=$ $\lambda_{\mathrm{g}} /(2 n)$, the size of the antenna can be significantly reduced via the enhanced refractive index of the MWS $n=\sqrt{\varepsilon_{\text {eff }} \mu_{\text {eff }}}$.

The simulated return loss of the MWS antenna with the effective parameters extracted in Fig. 4 compared with that of the homogeneous substrate antenna with same resonance frequency is presented in Fig. 8.

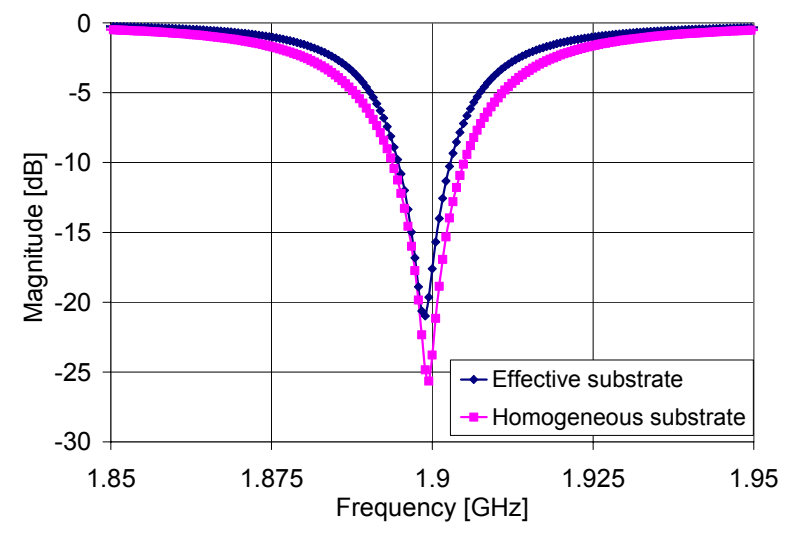

Figure 8. Simulated return loss: effective substrate with the effective parameters extracted in Fig. 4 compared with a homogeneous substrate $\left(\varepsilon_{r}=2.94\right)$.

The simulated radiation patterns of the MWS antenna compared with those of the homogeneous substrate antenna are shown in Fig. 9.

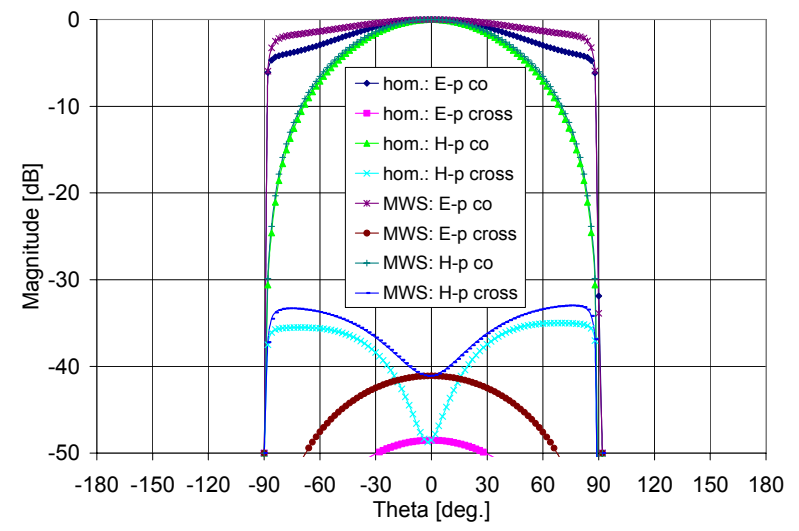


Figure 9. Simulated co and cross radiation patterns in the E-plane (E-p) and H-plane (H-p).

In Tab. 1, the principle parameters of the antenna are presented.

\begin{tabular}{|c|c|c|}
\hline $\mathrm{f}=1.9 \mathrm{GHz}$ & $\begin{array}{c}\text { MWS } \\
\left(\varepsilon_{\mathrm{eff}}=4.8,\right. \\
\left.\mu_{\mathrm{eff}}=1.3\right)\end{array}$ & $\begin{array}{c}\text { Homogeneous } \\
\text { substrate } \\
\left(\varepsilon_{\mathrm{r}}=2.94\right)\end{array}$ \\
\hline Dimension & $\mathrm{L}=32.3 \mathrm{~mm}$ & $\mathrm{~L}=46.3 \mathrm{~mm}$ \\
\hline Gain & $5.5 \mathrm{dBi}$ & $5.8 \mathrm{dBi}$ \\
\hline
\end{tabular}

Table 1. Parameters comparison for the MWS and homogeneous substrate antenna

The MWS microstrip patch antenna exhibits a $30 \%$ size (resonant length) reduction compared to the patch antenna on a conventional substrate, with similar return loss $(\sim 20 \mathrm{~dB})$ and co-cross polarization isolation $(\sim 40$ $\mathrm{dB}$ ). Tab. 1 shows that the size reduction (and subsequent directivity reduction due to reduced aperture) has not been done at the expense of significant gain reduction, which suggests that the efficiency of the homogeneous antenna could be preserved. This is most likely due to the fact that the permeability is increased at the same time as the permittivity, which keeps the input impedance of the antenna almost unchanged.

\section{CONCLUSION}

This paper has presented a characterization of the metallic wire substrate (MWS) in terms of its effective permittivity $\varepsilon_{e f f}$ and permeability $\mu_{e f f}$ and the application of the MWS to a microstrip patch antenna.

It has been shown by way of full-wave simulations that the MWS exhibits artificial magneto-dielectric properties with simultaneously enhanced effective permittivity and permeability over a large frequency band. An MWS microstrip line structure with values of $\varepsilon_{e f f}$ ranging from 4 to 9 and of $\mu_{\text {eff }}$ ranging from 1.3 to 2 , respectively, has been achieved. A size reduction of $30 \%$ without significant gain reduction has been achieved in the MWS microstrip antenna.

\section{ACKNOWLEDGEMENT}

I would like to thank Jules Gauthier, Roch Brassard and Samer Abielmona for their help in fabrication and measurements of the prototype.

\section{REFERENCES}

[1] C. Caloz, "Novel passive and active transmission line metamaterial devices", Proc. Symposium of the Materials Research Society (MRS), Spring Meeting, Workshop Negative Index Materials: From Microwave to Optical, San Francisco, CA, USA, CD-ROM, Invited talk, April 2006.

[2] R. E. Collin, Field Theory of Guided Waves, $2^{\text {nd }}$, New York: IEEE Press, 1991. (ch. 12).

[3] W. E. Kock, "Metallic delay lenses" Bell Syst. Tech. J., vol. 27, pp. 58-82, 1948.

[4] S. B. Cohn, "Analysis of the metal strip delay structure for microwave lenses" J. Appl. Phys., vol. 20, pp. 257-262, Mar. 1949.

[5] S. B. Cohn, "The electric and magnetic constants of metallic delay media contaning obstacles of arbitrary shape and thickness" J. Appl. Phys., vol. 22, pp. 628-634, May 1951.

[6] S. B. Cohn, "Microwave measurements on Metallic Delay Media” Proc. IRE, vol. 41, pp. 1177-1183, Sept. 1953.

[7] N. J. Kolettis and R. E. Collin, "Anisotropic properties of striptype artificial dielectric" IRE Trans. Microwave Theory Tech., vol. MTT-9, pp. 436-441, 1961.

[8] M.-K. Hu and D. K. Cheng, "A new class of artificial dielectrics," WESCON/58 Conf. Record, vol. 2, pp. 21-25, Aug. 1958.

[9] H.V. Nguyen, J. Gauthier, J.M. Fernandez, M.Sierra-Castañer and C. Caloz, "Metallic Wire Substrate (MWS) for Miniaturization in Planar Microwave Application", Asia-Pacific Microwave Conference APMC, Yokohama, Japan, December 2006.

[10] J. Machac, "Microstrip Line on an Artificial Dielectric Substrate”, IEEE Microwave and Wireless Components Letters, Vol. 16, N $\mathrm{N}^{\circ}$ 7, pp. 416-418, July 2006.

[11] H. Mossallaei and K. Sarabandi, "Magneto-Dielectrics in Electromagnetics: Concept and Applications", IEEE Trans. Antennas and Propagat., vol. 52, $\mathrm{N}^{\circ} 6$, pp. 1558-1567, June 2004.

[12] K. Buell, H. Mosallaei, K. Sarabandi, "A Substrate for Small Patch Antennas Providing Tunable Miniaturization Factors," IEEE Trans. Microwave Theory Tech., vol. 54, no. 1, pp. 135146, Jan 2006.

[13] D. M. Pozar, Microwave Engineering, $3^{\text {rd }}$ ed. Hoboken, NJ: J. Wiley, 2005.

[14] R.C. Hansen, "Fundamental Limitations in Antennas", Proc. IEEE 69, pp. 170-182, 1981. 